\title{
Ultralow-Noise Atomic-Scale Structures for Quantum Circuitry in Silicon
}

\author{
Saquib Shamim, ${ }^{\dagger}$ Bent Weber $,{ }^{\ddagger}, \S$ Daniel W. Thompson, ${ }^{\ddagger}$ Michelle Y.Simmons, ${ }^{\ddagger}$ and Arindam Ghosh, ${ }^{\dagger}$ \\ ${ }^{\dagger}$ Department of Physics, Indian Institute of Science, Bangalore 560 012, India \\ ${ }^{\ddagger}$ Centre for Quantum Computation and Communication Technology, \\ University of New South Wales, Sydney NSW 2052, Australia and \\ $\S$ School of Physics and Astronomy, Monash University, Melbourne VIC 3800, Australia
}

SUPPORTING INFORMATION

Fabrication of STM-patterned nanostructures

The atomic-scale wires (W1 and W2) have been patterned using STM lithography $[1,2]$ on the hydrogen terminated $\mathrm{Si}(001)-2 \times 1$ reconstructed surfaces of $\mathrm{n}$-type doped $(\mathrm{P}, 1-10 \mathrm{~m} \Omega \mathrm{cm})$ substrates. Following lithography, the wires were exposed to phosphine $\left(\mathrm{PH}_{3}\right)$ gas $\left(5 \times 10^{-8} \mathrm{mbar}, 6 \mathrm{~min}\right)$, passivating the reactive silicon dangling bonds and protecting the wire against contaminants during patterning of electrodes (S, D, V1, V2) and gates (G1, G2) connecting the wires to micrometer-scale Si:P doped contacts (See image in Figure S1a) $[1,2]$. A second exposure to $\mathrm{PH}_{3}$, followed by annealing $\left(350{ }^{\circ} \mathrm{C}, 1 \mathrm{~min}\right)$ and low-temperature silicon epitaxy $(\simeq 25 \mathrm{~nm})$, selectively dopes the completed pattern to density $\simeq 2 \times 10^{14} \mathrm{~cm}^{-2}$ with atomically sharp doping profiles and equivalent bulk density $\sim 10^{21} \mathrm{~cm}^{-3}$. The quantum dots (D1 and D2) were fabricated using the similar technique of STM lithography and subsequent silicon epitaxy. The quantum dot D2 has a surface gate on the silicon dioxide dielectric grown on the epitaxial silicon at $140{ }^{\circ} \mathrm{C}[3]$

\section{Measurement of low frequency noise}

An AC four probe technique has been used for noise measurements, in which a constant current was passed through the sample and the voltage was measured using the lock-in technique. The voltage fluctuations (in-phase X-component and out-of-phase Y-component) are recorded as a function of time using a 16-bit digitizer (Gage Compuscope 1600). The raw time series data is then digitally processed to obtain the power spectral density (PSD). The PSD of the Y-component is the background noise (Johnson's noise and amplifier noise) which is subtracted from the total noise (PSD of X-component) to obtain the noise from the sample, $S_{V}$. From $S_{V}$ and the known conductance $G, S_{G} / G^{2}$ can be calculated and integrated within the experimental bandwidth to obtain the variance of conductance fluctuations, $N_{G}=\left\langle\delta G^{2}\right\rangle /\left\langle G^{2}\right\rangle=\int S_{G} / G^{2} d f$. For the devices with very low conductance $\left(\ll e^{2} / h\right)$, a constant AC voltage was applied across the device and the current was measured using a current amplifier. The fluctuations in current were recorded and the PSD was calculated using the method described above.

\section{Conductance fluctuations in wire W2}

The scanning tunneling microscope image of the wire W2 is shown in Figure S1a. It has a diameter $\approx 4.6 \mathrm{~nm}$ and is $\approx 47 \mathrm{~nm}$ long. The conductance, $G$, as a function of gate voltage, $V_{g}$, for wire W2 at temperature $T=4.2 \mathrm{~K}$ is shown in Figure S1b. In the entire gate voltage range, the wire is metallic with $G>e^{2} / h$ and exhibits universal conductance fluctuations as has been shown previously [2]. The time series of conductance fluctuations at different gate voltages $(-0.7,-0.1$ and $0.8 \mathrm{~V})$ is shown in Fig. S1c and corresponding power spectral density (PSD), $S_{G} / G^{2}$, is shown in Figure S1d. Towards positive gate voltages (for example $V_{g}=0.8 \mathrm{~V}$ ), the time series appears to be random without jumps or switches (orange line in Figure S1c) and the corresponding PSD is $1 / f$ in nature as shown by orange triangles in Figure S1d. At negative gate voltages, the time series shows jumps in conductance which leads to the deviation of spectrum from $1 / f$ (dark green squares in Figure S1d).

\section{Gate voltage dependence of conductance fluctuations for wires W1 and W2}

The normalized variance of conductance fluctuations, $\left\langle\delta G^{2}\right\rangle /\left\langle G^{2}\right\rangle=\int S_{G} / G^{2} d f$, obtained by integrating the PSD over the experimental bandwidth has been compared with the transconductance, $d G / d V_{g}$, of the wires W1 and W2 
(a)

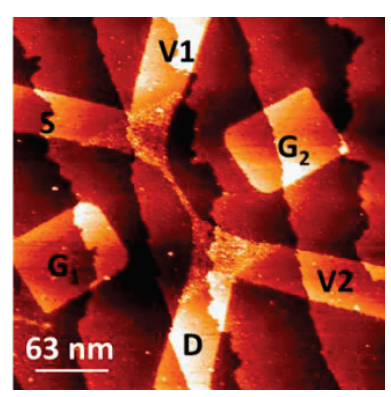

(b)

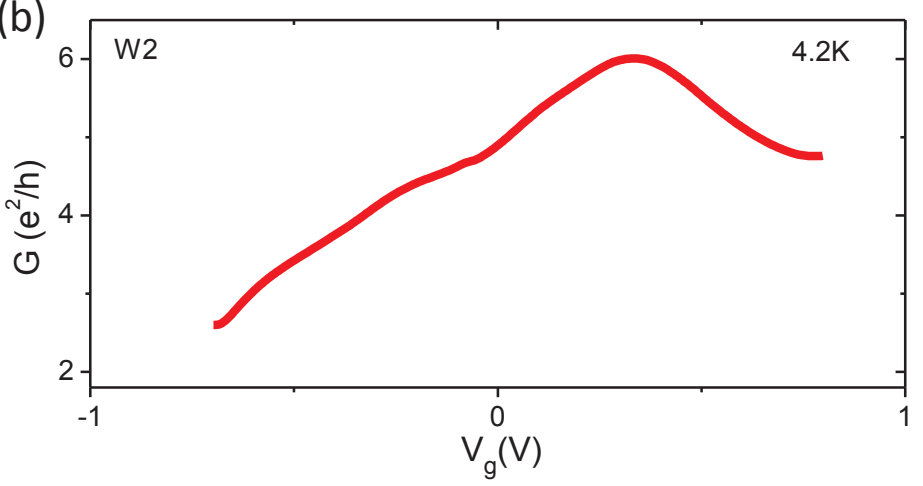

(c)

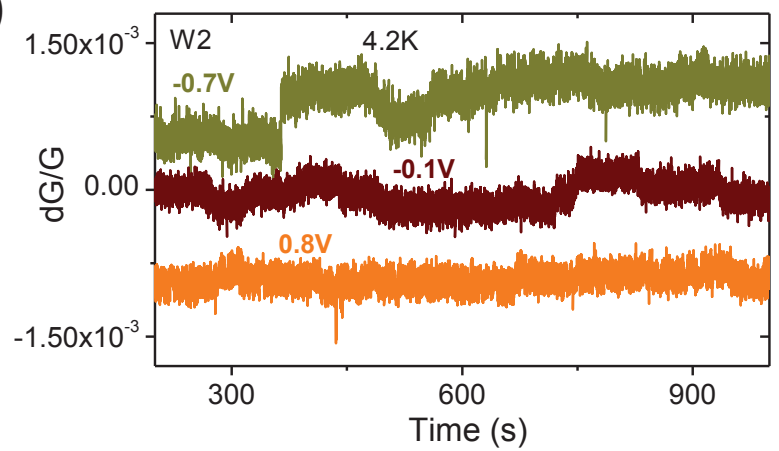

(d)

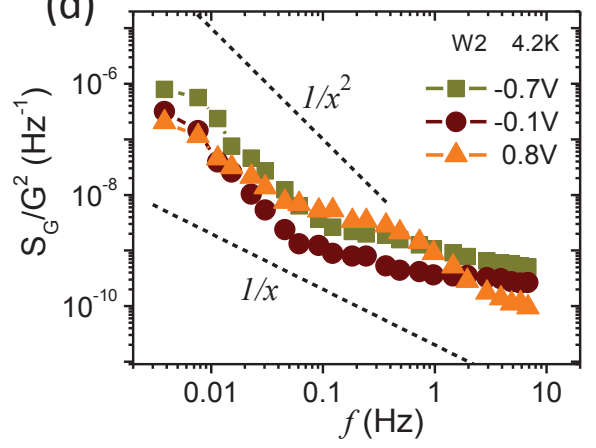

FIG. S1: Conductance fluctuations in wire W2. (a) Scanning tunneling microscope image of Wire W2 of diameter 4.6 nm. (b) The conductance, $G$, as a function of gate voltage, $V_{g}$, for Wire W2 at $4.2 \mathrm{~K}$. (c) The time-series of conductance fluctuations at different gate voltages for Wire W2 at $4.2 \mathrm{~K}$. (d) The power spectral density, $S_{G} / G^{2}$, obtained from the time series shown in (c).

in Figure S2a and S2b respectively. It can seen that $\left\langle\delta G^{2}\right\rangle /\left\langle G^{2}\right\rangle \propto\left(d G / d V_{g}\right)^{2}$ for the entire gate voltage range for both W1 and W2, indicating that the microscopic origin of conductance fluctuations is same for both the wires.

\section{Calculation of Hooge parameter}

In the main text, we have calculated the Hooge parameter by considering that fluctuations in conductance arise solely from the one-dimensional wire. The resistance of the two-dimensional region (2D) at the ends of the $1 \mathrm{D}$ wire is $R_{2 D} \sim 1900 \Omega$. The resistance of wire $\mathrm{W} 1$ is in the range $R_{W 1} \sim 20-200 \mathrm{k} \Omega$ while that of wire W2 is in the range $R_{W 2} \sim 5-12 \mathrm{k} \Omega$ at $4.2 \mathrm{~K}$. Since $R_{2 D} \ll R_{W 1, W 2}$, in the constant current measurement, most of the voltage drops across the $1 \mathrm{D}$ wire. Additionally, the dependence of the variance of conductance fluctuations $\left(\left\langle\delta G^{2}\right\rangle /\left\langle G^{2}\right\rangle\right)$ on transconductance $\left(d G / d V_{g}\right)$ also confirms that noise predominantly arises from the $1 \mathrm{D}$ wire since the transconductance of the $2 \mathrm{D}$ regions is virtually zero. Hence we calculated the Hooge parameter $\gamma_{H}^{W}$ of the wires as

$$
\gamma_{H}^{W}=\frac{S_{G}}{G^{2}} N f^{\alpha}
$$

where $\alpha$ is the frequency exponent and $N$ is the number of carriers. However, to be more precise, we do an exact calculation of the Hooge parameter of the wires by considering that the measured fluctuations is due to the entire system of 1D wires and 2D contact region. Considering $S_{V}^{T}$ to be the PSD of total measured voltage fluctuations (the system of $1 \mathrm{D}$ wire and the $2 \mathrm{D}$ regions at both the ends) and $S_{V}^{W}$ and $S_{V}^{2 D}$ to be the PSD of the voltage fluctuations in $1 \mathrm{D}$ wire and $2 \mathrm{D}$ regions respectively, we have

$$
S_{V}^{T}=S_{V}^{W}+S_{V}^{2 D}
$$



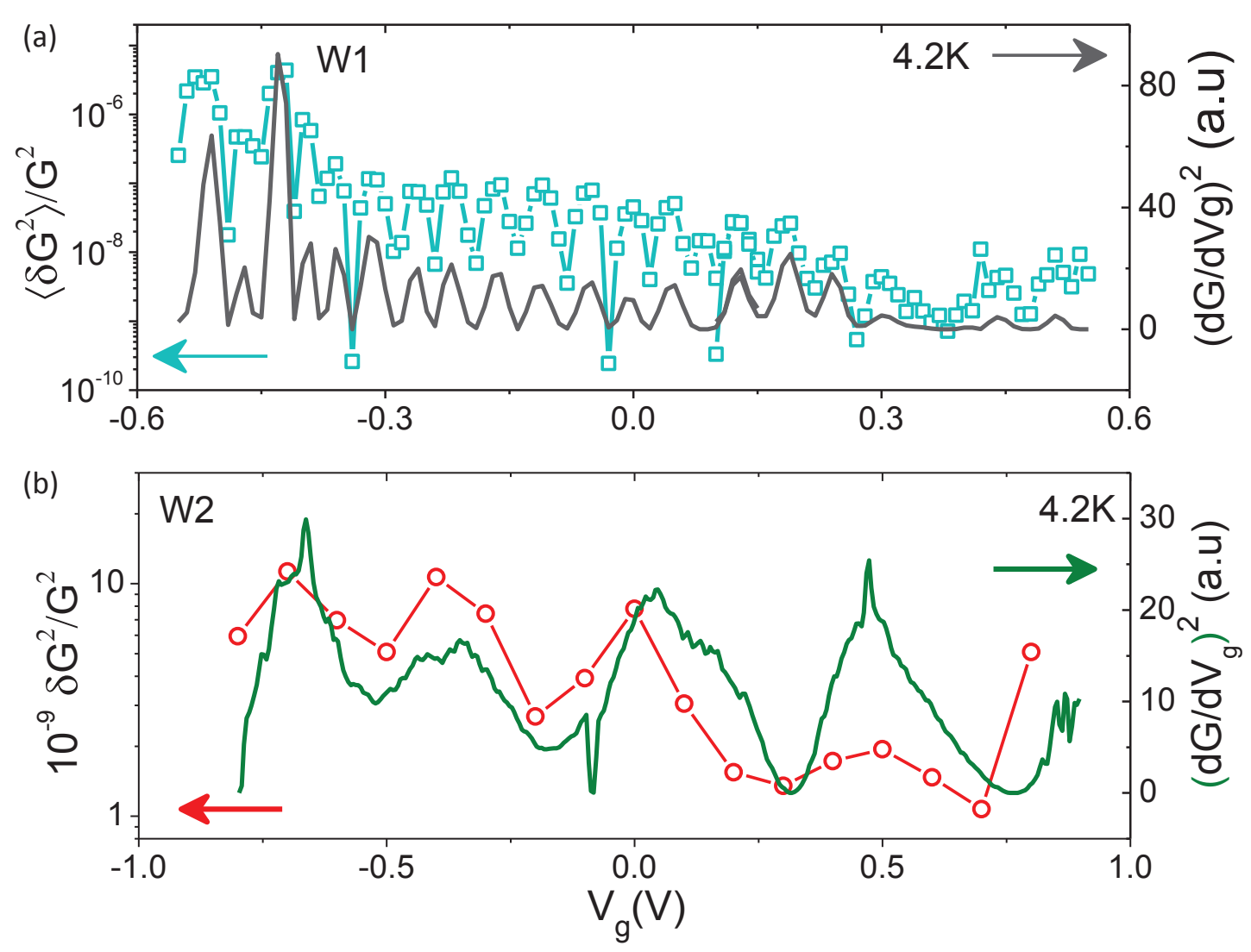

FIG. S2: Conductance fluctuations as a function of gate voltage The normalized variance, $\left\langle\delta G^{2}\right\rangle /\left\langle G^{2}\right\rangle=\int S_{G} / G^{2} d f$, as a function of gate voltage, $V_{g}$, for (a) Wire W1 and (b) Wire W2 at $4.2 \mathrm{~K}$. The axis on the right shows the dependence of square of transconductance $\left(d G / d V_{g}\right)^{2}$ on $V_{g}$.

Using the phenomenological Hooge's relation, $\gamma_{H}=S_{V} N f^{\alpha} / V^{2}$, we get

$$
S_{V}^{T}=\gamma_{H}^{W} \frac{V_{W}^{2}}{N_{W}} f^{-\alpha}+\gamma_{H}^{2 D} \frac{V_{2 D}^{2}}{N_{2 D}} f^{-\alpha}
$$

where $V_{W}$ and $V_{2 D}$ are the voltage bias across the $1 \mathrm{D}$ wire and $2 \mathrm{D}$ region respectively, while $N_{W}$ and $N_{2 D}$ are the total number of carriers in the $1 \mathrm{D}$ wire and the $2 \mathrm{D}$ region respectively. $\gamma_{H}^{W}$ and $\gamma_{H}^{2 D}$ represent the Hooge parameter of the $1 \mathrm{D}$ wire and the $2 \mathrm{D}$ region respectively. Since we are calculating $\gamma_{H}$ at $f=1 \mathrm{~Hz}$, the factor $f^{-\alpha}$ becomes unity. Rearranging, we get

$$
\gamma_{H}^{W}=\left(S_{V}^{T}-\gamma_{H}^{2 D} \frac{V_{2 D}^{2}}{N_{2 D}}\right) \frac{N_{W}}{V_{W}^{2}}
$$

In Eq. S4, $S_{V}^{T}$ has been measured experimentally, $V_{W}=I R_{W}$ and $V_{2 D}=I R_{2 D}$ where $I$ is the current through the device and $R_{W}$ and $R_{2 D}$ can be determined as outlined in Ref. [2]. The number of carriers in the wire is $N_{W}=n_{\delta} L W$ where $n_{\delta} \sim 2 \times 10^{14} \mathrm{~cm}^{-2}$ is the saturation doped density and $L$ and $W$ are the length and width of the wire respectively. Similarly, $N_{2 D}=n_{\delta} A_{2 D}$, where $A_{2 D}$ is the total area of the $2 \mathrm{D}$ region at both ends of the wire, can be calculated. For wire W1 we found that $N_{W 1} \approx 147$ and $N_{2 D} \sim 7600$ while for wire W2, $N_{W 2} \approx 432$ and $N_{2 D} \sim 10600$. The Hooge parameter of the $2 \mathrm{D}$ region was calculated from noise measurements in the saturation doped $2 \mathrm{D}$ Si:P $\delta$ layers and lies in the range $\gamma_{H}^{2 D} \sim 10^{-6}-10^{-5}[4,5]$. Substituting the values, the Hooge parameter of the $1 \mathrm{D}$ wire $\gamma_{H}^{W}$ was calculated using Eq. S4 and compared with the value evaluated using Eq. S1 in Figure S3a and S3b for wire W1 and W2 respectively. It is evident that for wire W1 the Hooge parameter calculated using Eq. S1 and Eq. S4 are identical while for wire W2, the they differ by a factor of $\sim 2-3$ towards positive gate voltages. This is due to 
(a)

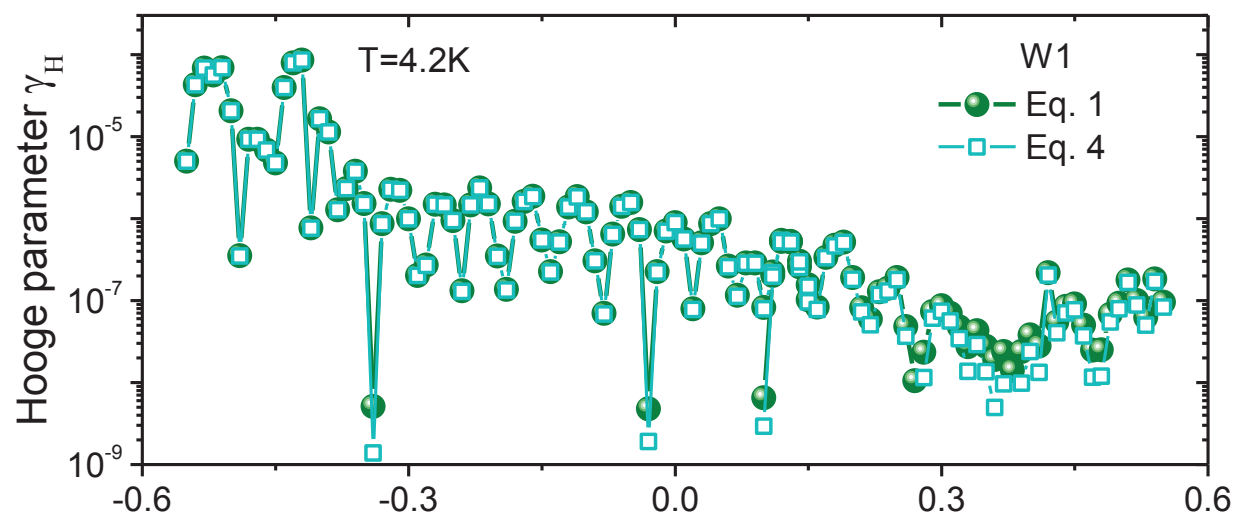

(b)

(c)
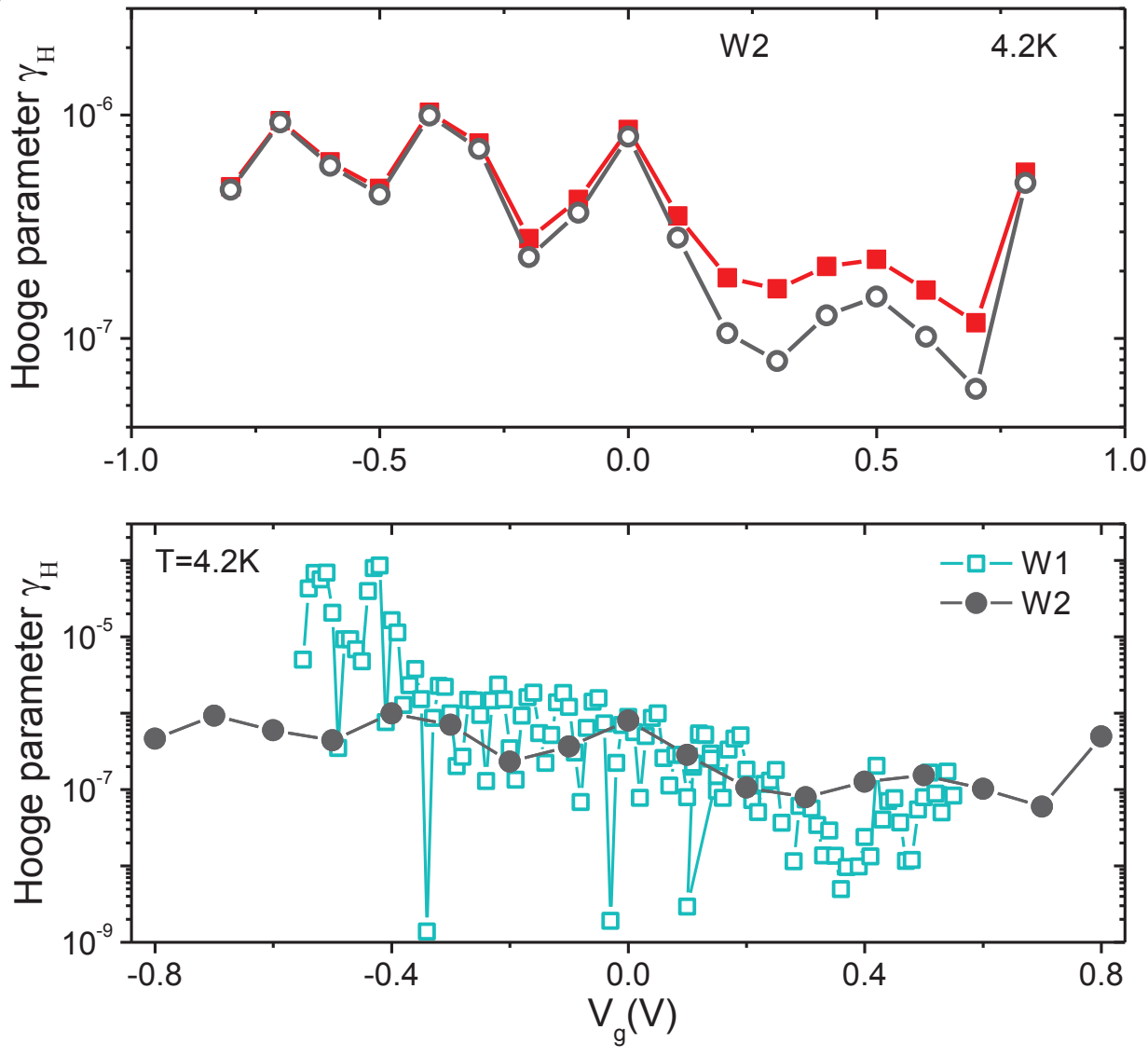

FIG. S3: Hooge parameter of 1D Si:P wires. Comparison of Hooge parameter calculated using Eq. S1 and Eq. S4 for (a) Wire W1 and (b) Wire W2 at 4.2 K. (c) Hooge parameter of W1 and W2 calculated using Eq. S4 plotted on the same scale.

appreciable voltage drop across the $2 \mathrm{D}$ contact region $\left(\sim 25 \%\right.$ of the total bias), whose resistance $R_{2 D} \approx 1.9 \mathrm{k} \Omega$ is not negligible as compared to $R_{W} \sim 5 \mathrm{k} \Omega$ at positive gate voltages. Nevertheless, the estimated Hooge parameter is one of the lowest reported for a $1 \mathrm{D}$ system.

The noise magnitude of the wire W1 varies by $\sim 4$ orders of magnitude (at negative gate voltage) as compared to wire W2 where it changes by an order of magnitude. However, at large positive gate bias - where both wires enter the metallic regime - the Hooge parameter is comparable as shown in Figure S3c. 


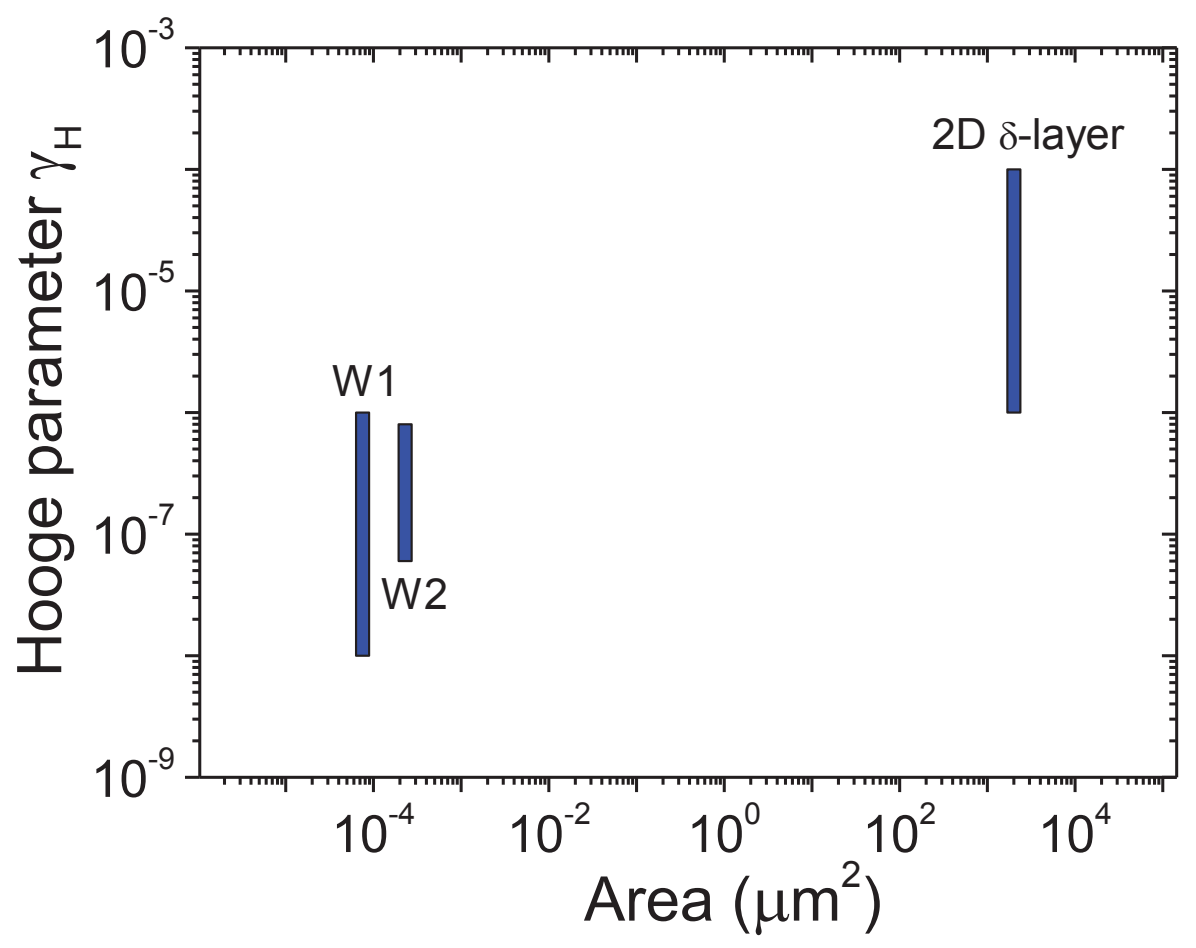

FIG. S4: Dimensionality dependence of Hooge parameter. The Hooge parameter, $\gamma_{H}$, as a function of planar area of the device.

\section{Comparison of Hooge parameter for 2D Si:P $\delta$-layers and $1 \mathrm{D}$ wires}

Figure S4 shows $\gamma_{H}$ as a function of the area of the device. We have included the 1D wires W1 and W2 and the 2D Si:P $\delta$-layers. For W1 and W2, here we have included the range of Hooge parameter in the positive gate voltage (i.e. metallic) regime. Upon breakdown of metallic conduction however, the onset of Coulomb blockade oscillations at negative gate voltages leads to regions of large transconductance and a concomitant increase in the Hooge parameter (that of W1 is even greater than the $2 \mathrm{D} \delta$-layers). The increased noise in $2 \mathrm{D}$ can be understood as due to influence of a large ensemble of charge traps. The correlation among charge traps would reduce beyond a distance $2 d(d$ is the thickness of epitaxial silicon) due to screening by the high density Si:P $\delta$-layer. Because of the large size of the 2D system, it is affected by the traps which are separated by distance greater than $2 d$ as opposed to the 1D wires which are affected only by a few fluctuators as discussed in Ref. [6].

\section{Charge noise in quantum dots D1 and D2}

We have estimated the power spectral density of charge fluctuations, $S_{Q}$, for quantum dots D1 and D2 as shown in Figure S5. For D1 we estimate $S_{Q} \sim 3 \times 10^{-9} \mathrm{e}^{2} \mathrm{~Hz}^{-1}$ while for D2 we get $S_{Q} \sim 10^{-8} \mathrm{e}^{2} \mathrm{~Hz}^{-1}$ at $f=1 \mathrm{~Hz}$ which compares favourably with the value of $S_{Q} \sim 3 \times 10^{-9} \mathrm{e}^{2} \mathrm{~Hz}^{-1}$ at $f=1 \mathrm{~Hz}$ in Ref. [7]. However, as a word of caution we would like to mention a few points. Firstly, the measurement bandwidth for the devices D1 and D2 $(1 \mathrm{mHz}-0.1 \mathrm{~Hz})$ is different from Ref. [7] $(0.2 \mathrm{~Hz}-100 \mathrm{~Hz})$. Hence we cannot directly compare the variance of charge fluctuations. For devices D1 and D2, we have estimated the value at $1 \mathrm{~Hz}$ by extrapolating the curve for $S_{Q}$ vs $f$ as shown in Figure S5 since our bandwidth was restricted to $0.1 \mathrm{~Hz}$. For both D1 and D2, we get $S_{Q} \propto 1 / f^{1.9}$, which is indicative of noise due to a single fluctuator as has also been observed Ref. [7]. However the corner frequency $f_{c}$ (below which the PSD tends to become flat) is very different for our devices D1 and D2 $(\sim 1-3 \mathrm{mHz})$ as compared to the devices in the Ref. [7] $\left(f_{c} \sim 7 \mathrm{~Hz}\right)$. These differences between the devices and the measurement bandwidth make the exact comparison difficult. Additionally we have not measured a detailed gate voltage dependence of noise in quantum dots D1 and D2. The main motivation of studying the quantum dots was to see the sensitivity of the device to the surface states which we did by recording successive gate voltage sweeps and measuring the horizontal 


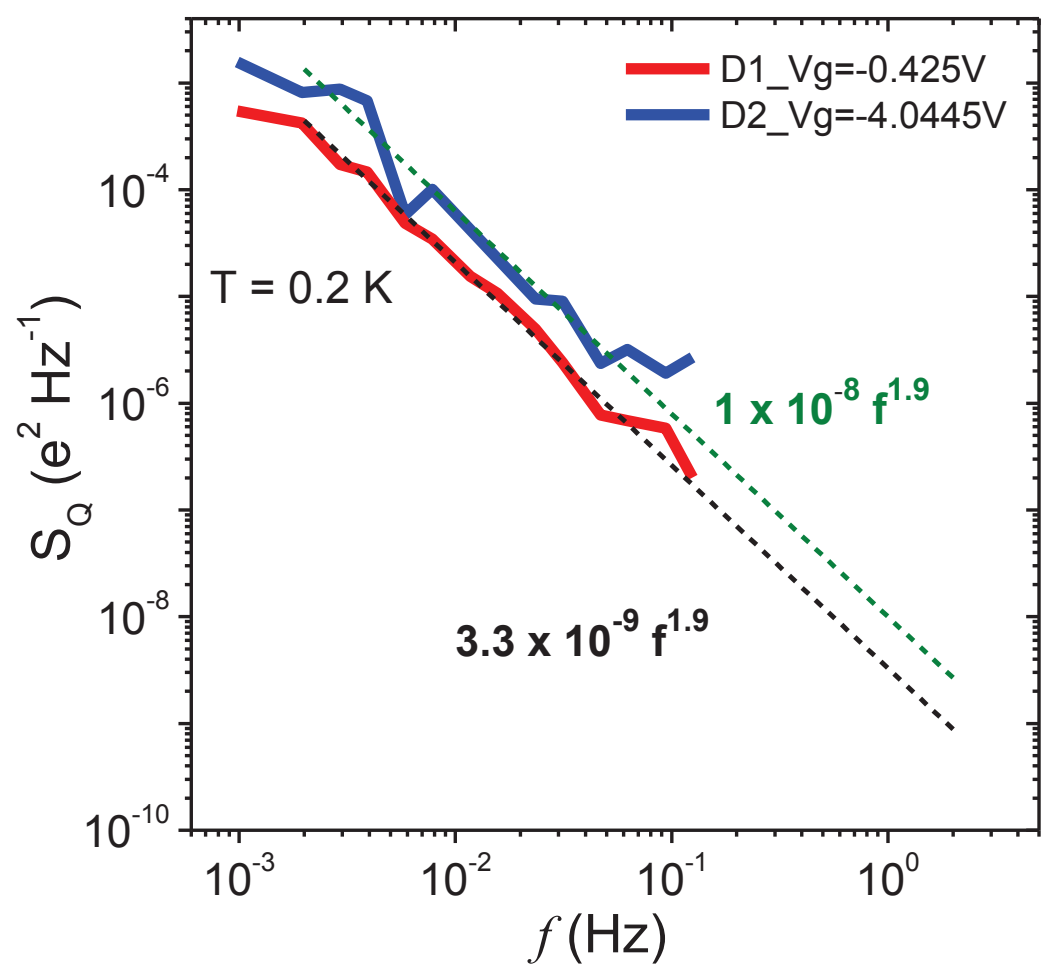

FIG. S5: Charge noise in quantum dots D1 and D2 The power spectral density of charge fluctuations, $S_{Q}$, as a function of frequency, $f$, for quantum dots D1 and D2 at temperature $0.2 \mathrm{~K}$. The dotted line shows $1 / f^{\alpha}$-type fits to the experimental points.

shift of Coulomb blockade peaks.

[1] Weber, B.; Mahapatra, S.; Ryu, H.; Lee, S.; Fuhrer, A.; Reusch, T. C. G.; Thompson, D. L.; Lee, W. C. T.; Klimeck, G.; Hollenberg, L. C. L.; Simmons, M. Y. Science 2012, 335, 64-67.

[2] Weber, B.; Ryu, H.; Tan, Y.-H. M.; Klimeck, G.; Simmons, M. Y. Phys. Rev. Lett. 2014, 113, 246802.

[3] Thompson, D. W. Ph.D. thesis, University of New South Wales, 2011.

[4] Shamim, S.; Mahapatra, S.; Polley, C.; Simmons, M. Y.; Ghosh, A. Phys. Rev. B 2011, 83, 233304.

[5] Shamim, S.; Mahapatra, S.; Scappucci, G.; Klesse, W. M.; Simmons, M. Y.; Ghosh, A. Phys. Rev. Lett. $2014,112,236602$.

[6] Clément, N.; Nishiguchi, K.; Fujiwara, A.; Vuillaume, D. Nat. Commun. 2010, 1, 92.

[7] Zimmerman, N. M.; Huber, W. H.; Simonds, B.; Hourdakis, E.; Fujiwara, A.; Ono, Y.; Takahashi, Y.; Inokawa, H.; Furlan, M.; Keller, M. W. J. Appl. Phys. 2008, 104, 033710. 